Arch. Tierz., Dummerstorf 43 (2000) 5, 451-461

Aus dem Institut fur Tierzucht und Tierhaltung der Christian-Albrechts-Universitat zu Kiel

SUSANNE KARSTEN, RAINER RÖHE, VOLKER SCHULZE, HOLGER LOOFT und ERNST KALM

\title{
Genetische Beziehungen zwischen individueller Futteraufnahme während der Eigenleistungsprüfung und Fruchtbarkeitsmerkmalen beim Schwein
}

Herrn Professor Dr. Dr. h.c. Gerhard von Lengerken zum 65. Geburtstag gewidmet

\begin{abstract}
Summary
Title of the paper: Genetic association between individual feed intake during performance test and reproductions traits in pigs

The present study deals with the estimation of genetic correlations between performance test traits measured in boars and reproduction traits of their offspring (sibs) of two purebred lines. Performance traits were individual feed intake (FI) recorded by electronic feeder, feed conversion ratio (FCR), average daily gain (ADG) and backfat thickness (BF). Reproduction traits were number of total piglets born (NBT) and born alive (NBA). Data of 4869 young boars, performance tested between April 1992 and May 1997 and data of 9710 primiparous sows, farrowed between June 1994 to November 1998, were observed. Heritabilities of performance traits recorded on station were in line 03 (line 04) $\mathrm{h}^{2}=0.24(0.33), 0.33(0.33), 0.23(0.32)$ and $0.47(0.53)$ for FI, ADG, FCR and $\mathrm{BF}$, respectively. Heritabilities of reproduction traits such as NBT, NBA were in line 03 (line 04) $h^{2}=0.05$ $(0.08)$ and $0.05(0.06)$, respectively. Genetic correlation between individual feed intake and reproduction traits ranged between $r_{8}=0.12$ and 0.27 . Undesirable genetic correlations were found between reproduction traits and feed efficiency $\left(r_{8}=0.13\right.$ to 0.44$)$, backfat thickness $\left(r_{8}=0.07\right.$ to 0.25$)$ and partly average daily gain $\left(r_{8}=-0.10\right.$ to 0.09 ). Line 04 showed lowest magnitude of feed intake and highest genetic antagonism between production and reproduction traits. It is suggested that feed intake capacity is a limiting factor for reproduction in line 04 and genetic antagonism between production and reproduction traits increases with reduction in feed intake.
\end{abstract}

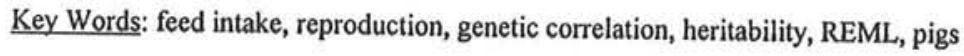

\section{Zusammenfassung}

Ziel der Untersuchung war die Schătzung der genetischen Korrelationen zwischen Produktionsmerkmalen von Ebern und Reproduktionsmerkmalen ihrer weiblichen Nachkommen fur zwei Reinzuchtlinien. Die Produktionsmerkmale setzten sich aus individuell erfasster Futteraufnahme (FUA), Futterverwertung (FVW), täglicher Zunahme (TZ) sowie Ruckenspeckdicke (RS) und die Reproduktionsmerkmale aus gesamt geborenen (GF) und lebend geborenen Ferkeln (LGF) zusammen. Als Datengrundlage dienten Eigenleistungsprufungen von 4869 Ebern der Jahre 1992 bis 1997 und die Wurfleistungen von 9710 Jungsauen in 1994 bis 1998. Die Heritabilitaten der Produktionsmerkmale der gepruften Eber betrugen in Linie 03 (Linie 04) fur die FUA, TZ, FVW und RS, $h^{2}$ $=0,24(0,33), 0,33(0,33), 0,23(0,32) 0,47(0,53)$. Für die GF wurden Heritabilităten in der Linie 03 (Linie 04 ) von $\mathrm{h}^{2}=0,05(0,08)$ und für die LGF von $\mathrm{h}^{2}=0,05(0,06)$ geschatzt. Die genetische Korrelation zwischen individueller FUA und Reproduktionsmerkmalen lag im Bereich von $r_{\mathrm{g}}=0,12$ bis 0,27 . Die Analysen ergaben züchterisch unerwilnschte Korrelationen zwischen Reproduktionsmerkmalen und FVW $\left(r_{8}=0,13\right.$ bis 0,44$)$, RS $\left(r_{g}=0,07\right.$ bis 0,25$)$ und teilweise zur TZ $\left(r_{g}=-0,10\right.$ bis 0,09$)$. Die Linie 04 mit der niedrigsten Futteraufnahme zeigte die hochsten antagonistischen genetischen Effekte zwischen Produktions- und Reproduktionsmerkmalen. Daraus lässt sich ableiten, dass in Linie 04 die Futteraufnahme ein limitierender Faktor fur Fruchtbarkeit ist und der Antagonismus wahrscheinlich ansteigt, wenn die Futteraufnahme weiter reduziert wird. Schlusselwörter: Futteraufnahme, Reproduktionsmerkmale, genetische Korrelation, Heritabilităt, REML, 


\section{Einleitung}

Die Selektion auf erhöhten Muskelfleischanteil hat aufgrund der negativen Beziehung zur Futteraufnahme zur Verminderung der Futteraufnahmekapazität beim Schwein geführt. Dadurch kann einerseits die tägliche Zunahme eingeschränkt werden und anderseits auch die Fruchtbarkeitsleistung von Jungsauen beeinträchtigt werden. Zudem zeigen Jungsauen in der Laktation eine große Varianz in der Futteraufnahme (CARROLL et al., 1996). Aufgrund der geringeren Körperreserven reagieren sie nach MULLAN und WILLIAMS (1989) empfindlicher auf eine geringe Futteraufnahme.

In der Literatur werden unterschiedliche Folgen einer geringen Futteraufnahme während der Laktation beschrieben. Zum einen weisen MULLAN und WILLIAMS (1989) darauf hin, dass eine hohe Futteraufnahme in der Laktation notwendig ist, um über eine hohe Milchleistung das Wachstumsvermögen der Ferkel auszuschöpfen. Zum anderen berichtet u.a. HUGHES (1989) von einem Einfluß der Futteraufnahme während der Laktation auf die Rastzeit und die folgende Wurfgröße.

Durch den Einsatz automatischer Futterstationen kann im Rahmen von Eigenleistungsprüfungen die tierindividuelle Futteraufnahme unter Gruppenhaltungsbedingungen erfasst werden. Die Schätzung der genetischen Beziehung der individuellen Futteraufnahme von Jungebern basierend auf elektronischen Futterstationen und der Fruchtbarkeit von weiblichen Nachkommen ist in der Literatur noch nicht analysiert worden. Außerdem ist von Interesse wie weitere Mastleistungsmerkmale wie tägliche Zunahme und Futterverwertung sowie das Schlachtkörperwertmerkmal Rückenspeckdicke von eigenleistungsgeprüften Jungebern zur Fruchtbarkeitsleistung der Nachkommen in genetischer Beziehung stehen. Auch sollte der Einfluß des Umfangs von Pedigreeinformationen auf die Schätzungen der genetischen Parameter untersucht werden.

\section{Material und Methoden}

Die Datengrundlage für die Schätzung der genetischen Beziehungen zwischen Produktions- und Fruchtbarkeitsmerkmalen bilden die Eigenleistungsprüfergebnisse von Ebern auf Station und die Fruchtbarkeitsergebnisse von Nachkommen, Voll- und Halbgeschwistern dieser Eber. Für die Analyse standen Mastleistungs- und Schlachtkörperwertinformationen von 4869 Jungebern, die im Zeitraum von April 1992 bis Mai 1997 in einer zentralen Eberteststation eigenleistungsgeprüft wurden, zur Verfügung. Die Tiere stammen aus zwei Reinzuchtlinien $03(n=2677)$ und $04(n=2192)$ und werden von vier Betrieben geliefert, wobei in jedem Betrieb beide Linien gehalten werden. Zunächst wurden die Tiere in einer zentralen Aufzuchtstation zur Quarantäne (ISOWEAN) vom 21. bis zum 90. Lebenstag gemeinsam aufgezogen. Danach erfolgte der eigentliche Stationstest im Alter vom 100. bis 170. Lebenstag in Gruppen von 12 Ebern pro Bucht. Die Eber wurden ad libitum an elektronischen Futterstationen der Herstellerfirma ACEMO gefüttert. Dadurch lag für jedes Tier die individuelle Futteraufnahme vor. Neben der Futteraufnahme je Tag wurden die tägliche Zunahme, Futterverwertung und die Rückenspeckdicke in die genetische Analyse aufgenommen. Die Mittelwerte und Standardabweichungen dieser Merkmale sind in Tabelle 1 aufgeführt. Die Probanden der Linie 03 zeigten eine um $44 \mathrm{~g}$ höhere tägliche Futter- 
aufnahme als die der Linie 04. Dies ergab eine um $22 \mathrm{~g}$ höhere tägliche Zunahme und eine um 0,17 mm dickere Rückenspeckdicke der Linie 03. Dagegen sind kaum Unterschiede in der Futterverwertung zwischen den Linien zu erkennen.

Tabelle 1

Mittelwerte ( $\bar{x})$ und Standardabweichungen (s) der Mastleistung und des Schlachtkörperwertes von Ebern der Linien 03 und 04 (Means $(\bar{x})$ and standard deviations (s) of fattening performances and backfat thickness of boars for lines 03 and 04)

\begin{tabular}{llcccc}
\hline Merkmal & & \multicolumn{2}{c}{ Linie 03 $(\mathrm{n}=2677)$} & \multicolumn{2}{c}{ Linie 04 (n=2192) } \\
& & $\overline{\mathrm{x}}$ & $\mathrm{s}$ & $\overline{\mathrm{x}}$ & $\mathrm{s}$ \\
\hline Anfangsgewicht & $(\mathrm{kg})$ & 46,1 & 5,5 & 45,2 & 5,4 \\
Endgewicht & $(\mathrm{kg})$ & 115,3 & 11,2 & 112,9 & 11,0 \\
Tăgliche Zunahme & $(\mathrm{g})$ & 1020 & 132 & 998 & 132 \\
Futteraufnahme & $(\mathrm{g})$ & 2555 & 368 & 2511 & 369 \\
Futterverwertung & $(\mathrm{kg} / \mathrm{kg})$ & 2,53 & 0,37 & 2,54 & 0,37 \\
Ruckenspeckdicke & $(\mathrm{mm})$ & 10,93 & 2,17 & 10,76 & 2,07 \\
\hline
\end{tabular}

Tabelle 2

Mittelwerte $(\bar{x})$ und Standardabweichungen (s) der gesamt geborenen und lebend geborenen Ferkel von Jungsauen in den Linien 03 und 04 (Means $(\bar{x})$ and standard deviations (s) of total born and born alive piglets of gilts for lines 03 and 04)

\begin{tabular}{|c|c|c|c|c|c|c|}
\hline \multirow[t]{2}{*}{ Merkmal } & \multicolumn{2}{|c|}{$\begin{array}{l}\text { Insgesamt } \\
(\mathrm{n}=9710)\end{array}$} & \multicolumn{2}{|c|}{$\begin{array}{c}\text { Linie 03 } \\
(\mathrm{n}=5089)\end{array}$} & \multicolumn{2}{|c|}{$\begin{array}{c}\text { Linie } 04 \\
(n=4621)\end{array}$} \\
\hline & $\overline{\mathrm{x}}$ & s & $\overline{\mathrm{x}}$ & s & $\bar{x}$ & s \\
\hline Gesamt geborene Ferkel/ Sau und Wurf & 10,4 & 2,9 & 10,2 & 2,9 & 10,5 & 2,9 \\
\hline Lebend geborene Ferkel/ Sau und Wurf & 9,6 & 2,8 & 9,6 & 2,8 & 9,7 & 2,7 \\
\hline
\end{tabular}

Die Fruchtbarkeitsleistungen von 9710 Sauen der Linien 03 (5089) und 04 (4621) wurden in der vorliegenden Analyse verwendet. Diese Sauen erbrachten im Zeitraum von Juni 1994 bis November 1998 ihren ersten Wurf in 10 Betrieben. Die Jungsauen wurden zu $75 \%$ künstlich besamt. Das mittlere Erstferkelalter betrug 345 Tage. Wie Tabelle 2 zeigt, unterscheiden sich die beiden untersuchten Linien kaum in der mittleren Wurfleistung. Die Überlegenheit der Linie 04 von 0,3 gesamt geborenen Ferkeln verminderte sich auf 0,1 lebend geborene Ferkel.

\section{Genetische Verknüpfung von Mastleistungs- und Fruchtbarkeitsinformationen}

Die genetische Verknüpfung der beiden Datensătze für Mastleistungsprufung sowie

Tabelle 3

Genetische Verknüpfung zwischen den Tieren mit Mastleistungs- oder Fruchtbarkeitsinformationen fur die Linie 03 (Genetic connectedness of animals with fattening or reproduction performances in line 03)

\begin{tabular}{lccc}
\hline & Mastleistungsprlfung (ZET) & Fruchtbarkeitsleistung & Insgesamt \\
\hline Probanden insgesamt & 2677 & 5089 & 7766 \\
Nachkommen von ZET-Ebern & - & 1328 & 1328 \\
\hline & \multicolumn{3}{c}{ Verwandte Tiere in beiden Datensätzen } \\
VG-Gruppen & 1,7 & & 343 \\
Probanden/ VG- Gruppe & 570 & 1,5 & 3,2 \\
Vollgeschwister & 12,0 & 513 & 1083 \\
\hline văterl. HG- Gruppen & 2129 & & 178 \\
Probanden/ HG- Gruppe & & 12,9 & 24,8 \\
văterliche Halbgeschwister & 2,3 & 2293 & 4422 \\
\hline multterl. HG-Gruppen & 1168 & 2,1 & 507 \\
Probanden/ HG- Gruppe & & 1074 & 4,4 \\
multterliche Halbgeschwister & & & 2242 \\
\hline
\end{tabular}

ZET $=$ Zentraler Ebertest; $V G=$ Vollgeschwister; $H G=$ Halbgeschwister 
Fruchtbarkeitsleistung ist vorwiegend über Nachkommen, Voll- und Halbgeschwister gegeben. In Linie 03 wurden insgesamt 7766 Probanden geprüft. Davon waren 1328 weibliche Nachkommen von eigenleistungsgeprüften Ebern, 1083 Tiere Vollgeschwister, 4422 väterliche und 2242 mütterliche Halbgeschwister (Tab. 3).

Wie in Tabelle 4 ersichtlich, werden 6813 insgesamt geprüfte Tiere der Linie 04 über 816 Nachkommen, 991 Vollgeschwister, 4072 väterliche und 2008 mütterliche Halbgeschwister genetisch verknüpft.

Tabelle 4

Genetische Verknupfung zwischen den Tieren mit Mastleistungs- oder Fruchtbarkeitsinformationen für die Linie 04 (Genetic connectedness of animals with fattening or reproduction performances in line 04)

\begin{tabular}{|c|c|c|c|}
\hline & Mastleistungsprufung (ZET) & Fruchtbarkeitsleistung & Insgesamt \\
\hline Probanden insgesamt & 2192 & 4621 & 6813 \\
\hline Nachkommen von ZET-Ebern & - & 816 & 816 \\
\hline & \multicolumn{3}{|c|}{ Verwandte Tiere in beiden Datensătzen } \\
\hline VG- Gruppen & & & 339 \\
\hline Probanden/ VG-Gruppe & 1,5 & 1,4 & 2,9 \\
\hline Vollgeschwister & 514 & 477 & 991 \\
\hline văterl. HG-Gruppen & & & 167 \\
\hline Probanden/ HG- Gruppe & 11,0 & 13,4 & 24,4 \\
\hline văterliche Halbgeschwister & 1843 & 2229 & 4072 \\
\hline mütterl. HG- Gruppen & & & 519 \\
\hline Probanden/ HG- Gruppe & 2,0 & 1,9 & 3,9 \\
\hline mütterliche Halbgeschwister & 1017 & 991 & 2008 \\
\hline
\end{tabular}

$\mathrm{ZET}=$ Zentraler Ebertest; VG= Vollgeschwister; $\mathrm{HG}=$ Halbgeschwister

\section{Statistische Modelle}

Die Varianzkomponentenschätzung erfolgte auf der Basis eines Tiermodells unter Verwendung der Methode REML, getrennt für die beiden Linien mit dem Programm VCE Version 4.2.5. von GROENEVELD (1998). Die Schätzung der genetischen Parameter für Futteraufnahme, Futterverwertung, tägliche Zunahme und Rückenspeckdicke wurden mit dem folgenden genetisch-statistischen Mehrmerkmalsmodell geschätzt.

$$
\begin{aligned}
& Y_{i j k l}=\mu_{1}+H_{i l}+S_{j l}+a_{i j k l}+b_{m l}\left(x_{i j k m}-\bar{x}_{m}\right)+e_{i j k l} \\
& Y_{\mathrm{ijkl}} \quad \text { Beobachtungswert des l-ten Merkmals } \quad(l=1, \ldots, 4)
\end{aligned}
$$

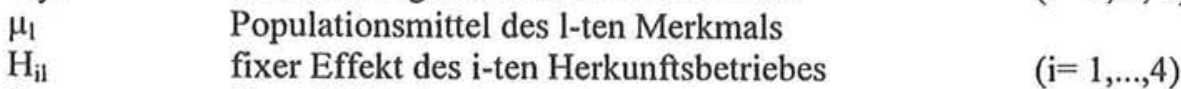

$$
\begin{aligned}
& \mathrm{S}_{\mathrm{jl}} \quad \text { fixer Effekt der j-ten Jahres-Saisonklasse } \quad(\mathrm{j}=1, \ldots, 16) \\
& \mathrm{a}_{\mathrm{ijkl}} \quad \text { zufälliger Tiereffekt des k-ten Tieres } \\
& b_{m l}\left(x_{i j k m}-\bar{x}_{m}\right) \text { lineare Regression auf die Abweichung des An- } \\
& \text { fangsgewichts }(m=1) \text {, Anfangsalters }(m=2) \text {, Endal- } \\
& \mathrm{e}_{\mathrm{ijkl}} \quad \text { Restfehler }
\end{aligned}
$$

Das Modell beinhaltet als systematische Einflußfaktoren den Herkunftsbetrieb der Eber und die Jahres-Saisonklasse bei Testbeginn (Quartalsklasse). Weiterhin werden die Kovariablen Anfangsgewicht, Anfangs- und Endalter berücksichtigt. Als zufällige Einflußfaktoren gehen der Tiereffekt und der Restfehler ein. 
Das Modell zur Schätzung der genetischen Parameter für die Fruchtbarkeitsmerkmale gesamt geborene und lebend geborene Ferkel berücksichtigt die nachfolgend aufgeführten Effekte

$$
\begin{aligned}
& Y_{i j k l m}=\mu_{m}+P_{i m}+L_{j m}+B S_{k m}+a_{i j k l m}+b_{m}\left(x_{i j k l}-\bar{x}\right)+e_{i j k l m} \\
& Y_{\mathrm{ijklm}} \quad \text { Beobachtungswert des m-ten Merkmals } \quad(m=1,2) \\
& \mu_{\mathrm{m}} \quad \text { Populationsmittel des m-ten Merkmals } \\
& \mathrm{P}_{\mathrm{im}} \quad \text { fixer Effekt der i-ten Paarungsart } \quad(\mathrm{i}=1,2) \\
& \mathrm{L}_{\mathrm{jm}} \quad \text { fixer Effekt der j-ten Linie des Belegebers } \quad(j=1,2) \\
& \mathrm{BS}_{\mathrm{km}} \quad \text { fixer Effekt der k-ten Betrieb-Jahres-Saisonklasse } \quad(\mathrm{k}=1, \ldots, 75)
\end{aligned}
$$

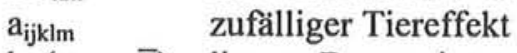

$$
\begin{aligned}
& b_{m}\left(x_{i j k l}-\bar{x}\right) \text { lineare Regression auf die Abweichung des Erstfer- } \\
& \text { kelalters } x_{i j k l} \text { vom mittleren Erstferkelalter } \bar{x} \\
& \mathrm{e}_{\mathrm{ijklm}} \quad \text { Restfehler }
\end{aligned}
$$

Dieses Modell beinhaltet als fixe Einflußfaktoren die Paarungsart (künstliche Besamung oder Natursprung), die Linie des Ebers, mit dem die Jungsau belegt wurde und den Betriebs-Jahres-Saisoneffekt, wobei jeweils 6 Monate eine Saisonklasse bilden. Zusätzlich wird eine Korrektur auf ein einheitliches Erstferkelalter vorgenommen.

\section{Ergebnisse und Diskussion}

\section{Einfluß des Umfangs von Pedigreeinformationen}

Die Schätzung der Heritabilitäten der Merkmale der Ebereigenleistungsprüfung auf Station wurde in zwei getrennten Analysen für Linie 03 und 04 durchgeführt. In der ersten Analyse wurden nur die elterlichen Abstammungen der Probanden verwendet, während in der zweiten Analyse die Pedigreeinformationen auf fünf Ahnengenerationen ausgedehnt wurden. Wie aus Tabelle 5 ersichtlich wird, kann die ausschließliche Berücksichtigung der elterlichen Abstammung zu einer Überschätzung der genetischen Parameter führen, während die Schätzfehler keine Abhängigkeit von der Tiefe des Pedigrees zeigen. In der Linie 03 sind die Schätzwerte bei ausschließlicher Berücksichtigung von elterlicher Verwandtschaft in den Merkmalen tägliche Zunahme und Futterverwertung um $18 \%$ beziehungsweise $17 \%$ höher als bei Verwendung des umfangreicheren Pedigrees. In Linie 04 ist ein größerer Einfluß des Umfangs an Pedigreeinformationen zu erkennen. Insbesondere bei der Futterverwertung erhöht sich die Heritabilität um 31\%, wenn das Pedigree auf nur elterliche Abstammung reduziert wird. Im Gegensatz zu Linie 03 beeinflußt der Umfang an Pedigreeinformationen in Linie 04 den Schätzwert für die Futteraufnahme. Die Veränderung der Schätzwerte verdeutlicht wie wichtig es ist, möglichst viele Pedigreeinformationen in die Varianzkomponentenschätzung einzubeziehen. Dies gilt insbesondere für Merkmale der Mastleistung wie Futteraufnahme, tägliche Zunahme und Futterverwertung, die eine niedrigere Heritabilität aufweisen als Schlachtkörperwertmerkmale wie Rückenspeckdicke, wo kaum Unterschiede in den Heritabilitäten zu verzeichnen waren. 
Tabelle 5

Einfluß des Umfangs an Pedigreeinformationen auf die Schätzung der Heritabilităten $\left(\mathrm{h}^{2}\right)$ der tăglichen $\mathrm{Zu}$ nahme (TZ), Futteraufnahme (FUA), Futterverwertung (FVW) und Rückenspeckdicke (RS) sowie deren Standardfehler $\left(\mathrm{s}_{\mathrm{h}}\right)$ fur die Linien 03 und 04 (Influence of the amount of pedigree information on estimation of heritabilities $\left(\mathrm{h}^{2}\right)$ of average daily gain (TZ), feed intake (FUA), feed conversion ratio (FVW) and backfat thickness (RS) for lines 03 and 04)

\begin{tabular}{|c|c|c|c|c|}
\hline \multirow{3}{*}{$\begin{array}{c}\text { Pedigree: } \\
\text { Anz. Tiere: } \\
\text { Merkmal }\end{array}$} & \multicolumn{2}{|c|}{ Linie 03} & \multicolumn{2}{|c|}{ Linie 04} \\
\hline & $\begin{array}{l}2 \text { Generationen } \\
4084\end{array}$ & $\begin{array}{l}5 \text { Generationen } \\
8654\end{array}$ & $\begin{array}{c}2 \text { Generationen } \\
3525\end{array}$ & $\begin{array}{c}5 \text { Generationen } \\
6963\end{array}$ \\
\hline & $h^{2} \pm s_{h^{2}}$ & $h^{2} \pm s_{h^{2}}$ & $h^{2} \pm s_{h^{2}}$ & $h^{2} \pm s_{h^{2}}$ \\
\hline RS & $0,45 \pm 0,04$ & $0,47 \pm 0,04$ & $0,51 \pm 0,04$ & $0,53 \pm 0,05$ \\
\hline $\mathrm{TZ}$ & $0,39 \pm 0,04$ & $0,33 \pm 0,04$ & $0,36 \pm 0,04$ & $0,33 \pm 0,04$ \\
\hline FUA & $0,25 \pm 0,03$ & $0,24 \pm 0,03$ & $0,40 \pm 0,03$ & $0,33 \pm 0,04$ \\
\hline FVW & $0,27 \pm 0,03$ & $0,23 \pm 0,03$ & $0,42 \pm 0,05$ & $0,32 \pm 0,05$ \\
\hline
\end{tabular}

\section{Genetische Parameter der Mastleistungs- und Schlachtkörperwertmerkmale}

In alle nachfolgenden genetischen Analysen gehen die Pedigreeinformationen aus den letzten fünf Ahnengenerationen ein. Die Schätzwerte der additiv genetischen Varianzen $\left(\hat{\sigma}^{2}\right)$, der Restvarianzen $\left(\hat{\sigma}_{\mathrm{e}}{ }_{\mathrm{e}}\right)$ und der Heritabilitäten $\left(\mathrm{h}^{2}\right)$ für die Merkmale der Mastleistung und des Schlachtkörperwertes werden in Tabelle 6 wiedergegeben. Die additiv genetischen Varianzen sind bei den Mastleistungsmerkmalen in der Linie 04 jeweils größer als in der Linie 03. Demgegenüber zeigt die Rückenspeckdicke der Linie 04 eine etwas niedrigere additiv genetische Varianz. Die Restvarianzen der untersuchten Merkmale sind bis auf die der täglichen Zunahme niedriger für Linie 04 als für Linie 03.

Tabelle 6

Additiv genetische Varianzen $\left(\hat{\sigma}_{\mathrm{a}}^{2}\right)$, restbedingte Varianzen $\left(\hat{\sigma}_{\mathrm{e}}\right)$ und Heritabilităten $\left(\mathrm{h}^{2}\right)$ in den Merkmalen Rückenspeckdicke (RS), tägliche Zunahme (TZ), Futteraufnahme (FUA) und Futterverwertung (FVW) in den Linien 03 und 04 (Additive genetic variances $\left(\hat{\sigma}_{n}\right)$, residual variances $\left(\hat{\sigma}_{e}\right)$ and heritabilities $\left(h^{2}\right)$ of backfat thickness (RS), average daily gain (TZ), feed intake (FUA) and feed efficiency (FVW) for lines 03 and 04)

\begin{tabular}{lcccccc}
\hline \multirow{2}{*}{ Merkmal } & \multicolumn{3}{c}{ Linie 03 $(\mathrm{n}=8654)$} & \multicolumn{3}{c}{ Linie 04 (n=6963) } \\
& $\hat{\sigma}^{2}{ }_{\mathrm{a}}$ & $\hat{\sigma}^{2}{ }_{\mathrm{e}}$ & $\mathrm{h}^{2} \pm \mathrm{s}_{\mathrm{h}^{2}}$ & $\hat{\sigma}^{2}{ }_{\mathrm{a}}$ & $\hat{\sigma}^{2}{ }_{\mathrm{e}}$ & $\mathrm{h}^{2} \pm \mathrm{s}_{\mathrm{h}^{2}}$ \\
\hline RS & 1,57 & 1,74 & $0,47 \pm 0,04$ & 1,53 & 1,38 & $0,53 \pm 0,05$ \\
TZ & 4754 & 9662 & $0,33 \pm 0,04$ & 4887 & 10039 & $0,33 \pm 0,04$ \\
FUA & 26959 & 84250 & $0,24 \pm 0,03$ & 36276 & 73459 & $0,33 \pm 0,04$ \\
FVW & 25179 & 85387 & $0,23 \pm 0,03$ & 36095 & 77044 & $0,32 \pm 0,05$ \\
\hline
\end{tabular}

$s_{\mathrm{b}}$ : Standardfehler der Heritabilităt

Die Schätzwerte für die Heritabilitäten der Mastleistungsmerkmale liegen in beiden Linien im mittleren Bereich von $\mathrm{h}^{2}=0,23$ bis 0,33 . In Linie 04 ergeben sich jedoch höhere Heritabilitäten für die Futteraufnahme und die Futterverwertung als in Linie 03, während die Heritabilitäten der täglichen Zunahme in beiden Linien ein gleiches Niveau aufweisen. Die Schätzwerte der Heritabilitäten und deren Niveauunterschiede zwischen Mastleistungs- und Schlachtkörperwertmerkmalen sind im Rahmen der in der Literatur angegebenen Bereiche (VON FELDE et al., 1996; VAN STEENBERGEN et al., 1990; MRODE und KENNEDY, 1993).

In beiden Linien werden die höchsten genetischen Korrelationen zwischen den Merkmalen Futteraufnahme und tägliche Zunahme geschätzt. Die geschätzten genetischen 
Beziehungen dieser Merkmale $\left(r_{g}=0,56\right.$ und 0,60$)$ liegen auf dem Niveau der von VAN STEENBERGEN et al. (1990) $r_{\mathrm{g}}=0,57$, CAMERON et al. (1990) $r_{\mathrm{g}}=0,58$ und VON FELDE et al. (1996) $r_{\mathrm{g}}=0,62$ geschätzten Werte. Demgegenüber berichten MRODE und KENNEDY (1993) und LABROUE et al. (1996) von höheren genetischen Korrelationen im Bereich von $r_{g}=0,80$ und 0,87 . Die züchterisch unerwünschte genetische Korrelation zwischen der Futteraufnahme und der Rückenspeckdicke entspricht der Erwartung aufgrund der bereits in diesem Abschnitt erwähnten Literaturquellen. Positive genetische Korrelationen zwischen Futteraufnahme und Futterverwertung werden auch von VAN STEENBERGEN et al. (1990) $r_{g}=0,33$ und CAMERON et al. (1990) $r_{g}=0,30$ analysiert, während LABROUE et al. (1996) und VON FELDE (1996) genetische Korrelationen im Bereich von Null angeben. Über die Richtung der genetischen Korrelationen zwischen Futterverwertung und Rückenspeckdicke kann keine Aussage getroffen werden, da diese für beide Linien nicht signifikant sind.

Tabelle 7

Genetische Korrelationen $\left(\mathrm{r}_{\mathrm{g}}\right)$ zwischen Ruckenspeckdicke (RS), tăgliche Zunahme (TZ), Futteraufnahme (FUA) und Futterverwertung (FVW) in den Linien 03 ( $n=8654$; oberhalb der Diagonalen) und $04(n=6963$; unterhalb der Diagonalen) (Genetic correlations $\left(r_{g}\right)$ among backfat thickness (RS), average daily gain (TZ), feed intake (FUA) and feed efficiency (FVW) for lines 03 (above diagonal) and 04 (below diagonal))

\begin{tabular}{|c|c|c|c|c|}
\hline Merkmal & $\begin{array}{c}\text { RS } \\
r_{8} \pm s_{r}\end{array}$ & $\begin{array}{c}\mathrm{TZ} \\
\mathrm{r}_{\mathrm{g}} \pm \mathrm{s}_{\mathrm{r}}\end{array}$ & $\begin{array}{c}\text { FUA } \\
r_{8} \pm s_{r}\end{array}$ & $\begin{array}{r}\text { FVW } \\
r_{g} \pm s_{t}\end{array}$ \\
\hline RS & & $0,53^{\circ} \pm 0,06$ & $0,46^{\circ} \pm 0,08$ & $-0,13 \pm 0,09$ \\
\hline $\mathrm{TZ}$ & $0,39^{\circ} \pm 0,07$ & & $0,60^{\circ} \pm 0,07$ & $-0,55^{\circ} \pm 0,07$ \\
\hline FUA & $0,52^{\circ} \pm 0,07$ & $0,56^{\circ} \pm 0,08$ & & $0,34^{\circ} \pm 0,09$ \\
\hline FVW & $0,13 \pm 0,10$ & $-0,45^{\circ} \pm 0,09$ & $0,48^{\circ} \pm 0,08$ & \\
\hline
\end{tabular}

$s_{\mathrm{f}}$ : Standardfehler der Kortelation: * signifikant $(\mathrm{p}<0,05)$

\section{Genetische Parameter der Fruchtbarkeitsmerkmale}

In Übereinstimmung mit der Literatur (ROEHE und KENNEDY, 1995; FISCHER et al., 1999; TÄUBERT und BRANDT, 2000) ergeben die Schätzungen für die Fruchtbarkeitsmerkmale gesamt geborene und lebend geborene Ferkel des ersten Wurfes nur niedrige Heritabilitäten (Tab. 8). Dabei erweist sich das Merkmal gesamt geborene Ferkel mit $h^{2}=0,05$ in beiden Linien als geringer erblich als das Merkmal lebend geborene Ferkel, das in Linie 03 eine Heritabilität von $h^{2}=0,08$ und in Linie 04 von $h^{2}=$ 0,06 aufweist.

Tabelle 8

Additiv genetische Varianzen $\left(\hat{\sigma}_{\mathrm{a}}\right)$, restbedingte Varianzen $\left(\hat{\sigma}_{\mathrm{c}}\right)$ und Heritabilităten $\left(\mathrm{h}^{2}\right)$ der Merkmale gesamt geborene (GF) und lebend geborene Ferkel (LGF) in den Linien 03 und 04 (Additive genetic variances $\left(\hat{\sigma}_{a}\right)$, residual variances $\left(\hat{\sigma}_{e}\right)$ and heritabilities $\left(h^{2}\right)$ of total born (GF) and born alive piglets (LGF) for lines 03 and 04$)$

\begin{tabular}{lccccccc}
\hline \multirow{2}{*}{ Merkmal } & \multicolumn{3}{c}{ Linie 03 $(\mathrm{n}=5089)$} & \multicolumn{3}{c}{ Linie 04 (n=4621) } \\
\hline GF & $\hat{\sigma}_{\mathrm{n}}^{2}$ & $\hat{\sigma}_{\mathrm{c}}$ & $\mathrm{h}^{2} \pm \mathrm{s}^{2}$ & $\hat{\sigma}_{\mathrm{s}}$ & $\hat{\sigma}_{c}$ & $\mathrm{~h}^{2} \pm \mathrm{s}_{\mathrm{h}^{2}}$ \\
LGF & 0,43 & 7,73 & 0,05 & 0,01 & 0,41 & 7,65 & $0,05 \pm 0,01$ \\
\hline
\end{tabular}

$s_{\mathrm{n}}$ : Standardfehler der Heritabilitat

\section{Genetische Korrelationen zwischen Mastleistung und Fruchtbarkeit}

Das wesentliche Ziel dieser Untersuchung war es, die genetischen Korrelationen zwi- 
schen der individuellen Futteraufnahme der eigenleistungsgeprüften Eber und der Fruchtbarkeitsleistung der weiblichen Nachkommen bzw. Geschwister zu schätzen. Dabei zeigte sich für die Linie 03 eine signifikante genetische Beziehung der Futteraufnahme zu den gesamt geborenen Ferkeln $\left(r_{g}=0,23\right)$. Demgegenüber ist in dieser Linie die genetische Beziehung zwischen Futteraufnahme und lebend geborene Ferkel mit $r_{g}=0,13$ nicht signifikant von Null verschieden. Im Vergleich zur Linie 03 wurden für Linie 04 höhere genetische Korrelationen zwischen Futteraufnahme und Fruchtbarkeitsmerkmalen $\left(r_{\mathrm{g}}=0,27\right)$ geschätzt. Diese Linie 04 zeigt auch die geringere mittlere Futteraufnahme von $998 \mathrm{~g}$ gegenüber $1020 \mathrm{~g}$ der Linie 03, wodurch indirekt abgeleitet werden kann, dass in der Linie 04 die Futteraufnahmekapazität wahrscheinlich eher eine Limitierung für die Fruchtbarkeitsleistung darstellt als in Linie 03. Die von CRUMP et al. (1997) geschätzten genetischen Korrelationen zwischen der Futteraufnahme und den lebend geborenen Ferkeln weisen mit $r_{g}=0,20$ ein ähnliches Niveau auf wie in der vorgestellten Untersuchung.

In beiden Linien wurden geringe negative genetische Korrelationen zwischen der täglichen Zunahme und den lebend geborenen Ferkeln geschätzt, die aber signifikant von Null abweichen. Eine ähnliche geringe negative genetische Korrelation zwischen diesen Merkmalen $\left(r_{g}=-0,08\right)$ wurde von CRUMP et al. (1997) geschätzt, die jedoch mit einem hohen Standardfehler von 0,14 angegeben wird. Zwischen der täglichen Zunahme und den gesamt geborenen Ferkeln konnten auch in der vorliegenden Untersuchung keine signifikanten genetischen Beziehungen festgestellt werden. Für dieses Merkmalspaar schätzten RYDHMER et al. (1992) eine höhere negative genetische Korrelation von $r_{g}=-0,14$.

Zwischen der Futterverwertung und der Anzahl der lebend geborenen Ferkel besteht in der Linie 03 eine züchterisch unerwünschte positive Beziehung, d.h. ein geringer Futterverbrauch je $\mathrm{kg}$ Zuwachs resultierte in eine geringe Wurfgröße. Wiederum in der Linie 04 werden höhere genetische Korrelationen zwischen der Futterverwertung und den Fruchtbarkeitsmerkmalen geschätzt. Insbesondere die genetische Korrelation der Futterverwertung zu den lebend geborenen Ferkeln weist mit $r_{g}=0,44$ ein mittleres Niveau auf. Dies kann wiederum andeuten, dass in der Linie 04 die Futteraufnahme als ein Faktor der Futterverwertung zu gering ist und somit zu einer unerwünschten genetischen Beziehung zwischen Futterverwertung und Fruchtbarkeitsleistung füht.

Tabelle 9

Genetische Korrelationen $\left(r_{8}\right)$ zwischen Ruckenspeckdicke (RS), tăgliche Zunahme (TZ), Futteraufnahme (FUA), Futterverwertung (FVW) und den gesamt geborenen (GF) sowie lebend geborenen Ferkeln (LGF) in den Linien 03 und 04 (Genetic correlations $\left(r_{8}\right)$ for backfat thickness (RS), average daily gain (TZ), feed intake (FUA) and feed conversion ratio (FVW) with total born (GF) and born alive piglets (LGF) in lines 03 and 04)

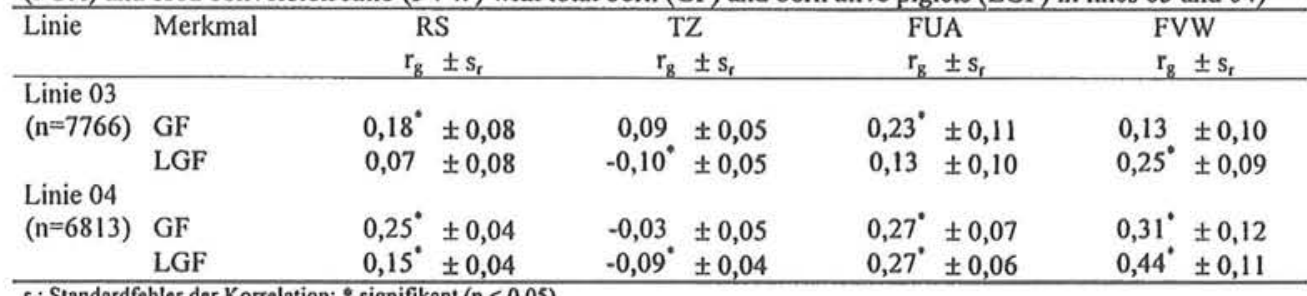

$s_{t}:$ Standardfehler der Korrelation; ${ }^{\circ}$ signifikant $(\mathrm{p}<0,05)$

Die Rückenspeckdicke ist in beiden Linien vorwiegend mit den gesamt geborenen Fer- 
keln genetisch korreliert $\left(r_{g}=0,18\right.$ in Linie 03 und $r_{g}=0,25$ in Linie 04). Für die Linie 04 wird weiterhin eine signifikante genetische Korrelation von $r_{g}=0,15$ mit der Anzahl lebend geborener Ferkel geschätzt. Die Linie 04 weist die geringere Rückenspeckdicke mit 10,76 mm im Vergleich zu 10,93 mm der Linie 03 auf, woraus abgeleitet werden kann, dass in Linie 04 womöglich die optimale Speckdicke für Fruchtbarkeitsleistungen von zahlreichen weiblichen Nachkommen unterschritten wird. Auf eine züchterisch unerwünschte Korrelation zwischen Rückenspeckdicke und Wurfgröße weisen bereits JOHANSSON und KENNEDY (1983) hin. Sie schätzten für Landrasse- und Yorkshire-Sauen genetische Korrelationen von $r_{g}=0,17$ bzw. $r_{g}=0,24$ zwischen Rückenspeckdicke und der Anzahl gesamt geborener Ferkel. Für die genetische Korrelation zwischen Rückenspeckdicke und der Anzahl lebend geborener Ferkel geben dieselben Autoren in Übereinstimmung mit der vorliegenden Untersuchung etwas geringere Werte an $\left(\mathrm{r}_{\mathrm{g}}=0,13\right.$ und 0,22$)$. Auch CRUMP et al. (1997) finden eine unerwünschte genetische Korrelation $\left(r_{\mathrm{g}}=0,21\right)$ zwischen Rückenspeckdicke und Anzahl lebend geborener Ferkel.

\section{Schlußfolgerungen}

Die Ergebnisse dieser Untersuchung zeigen, dass in beiden untersuchten Linien genetische Beziehungen zwischen der Mastleistung bzw. dem Schlachtkörperwert von Ebern und der Fruchtbarkeit ihrer weiblichen Nachkommen (Geschwister) bestehen. Grundsätzlich läßt sich aus den geschätzten genetischen Korrelationen ableiten, dass die traditionelle Selektion auf erhöhte tägliche Zunahme, verbesserte Futterverwertung und verminderte Rückenspeckdicke zu einer verminderten Fruchtbarkeitsleistung führt. Dabei zeigt neben der individuellen Futteraufnahme vor allem die Futterverwertung eine antagonistische Korrelation zur Wurfgröße. Zudem konnten Linienunterschiede in den genetischen Korrelationen zwischen Produktions- und Reproduktionsleistung festgestellt werden. So zeigt die Linie 04 höhere antagonistische Beziehungen als die Linie 03. Die Linie 04 weist zudem eine geringere mittlere Futteraufnahme und Rückenspeckdicke als Linie 03 auf, so dass angenommen werden kann, dass einige Tiere der Linie 04 das Optimum hinsichtlich Futteraufnahmekapazität und Rückenspeckdicke zur Erzielung einer hohen Fruchtbarkeit unterschritten haben. Zudem zeigt die Linie 04 die höheren antagonistischen Beziehungen zwischen Futteraufnahme und Rückenspeckdicke sowie Futterverwertung, so dass eine weitere Zucht auf verminderte Ruckenspeckdicke und verbesserte Futterverwertung die Futteraufnahme gravierend vermindert wird. Denn auf der Grundlage der vorliegenden Ergebnisse ist eine ausreichende Futteraufnahme nicht nur Voraussetzung für eine hohe tägliche Zunahme, sondern auch für eine hohe Fruchtbarkeit.

Außerdem zeigen die Untersuchungsergebnisse, dass die mit elektronischen Futterautomaten individuell erfasste Futteraufnahme von Vätern eine positive genetische Beziehung zur Wurfgröße ihrer Nachkommen aufweist. Somit wird sich eine Vernachlässigung der Futteraufnahme bei Selektionsentscheidungen nachteilig auf zukünftige Fruchtbarkeitsleistungen auswirken. Zudem geben KERR und CAMERON (1995) eine positive genetische Korrelation von $r_{g}=0,45$ zwischen Futteraufnahme während der Eigenleistungsprüfung und dem Wurfgewicht der Sauen bei Geburt und beim Absetzen an, so dass auch das Wachstum der Ferkel durch Selektion auf hohe 
Futteraufnahme verbessert wird. Entscheidend wäre noch zu prüfen, ob das Ferkeleinzelgewicht durch die Selektion auf höhere Futteraufnahme verbessert wird, da dieses Gewicht der weitaus bedeutendste Risikofaktor für die Überlebensfähigkeit von Ferkeln während der Säugeperiode ist (ROEHE und KALM, 2000).

Vor allem bei Jungsauen und Sauen mit großen Würfen reicht die Futteraufnahme in der Laktation häufig nicht aus, um den erhöhten Nährstoffbedarf zu decken. Eine hohe Futteraufnahme in der Laktation ist jedoch Voraussetzung für eine hohe Milchleistung in der späten Laktation, ohne die das Wachstumsvermögen der Ferkel nicht voll ausgeschöpft werden kann (MULLAN und WILLIAMS, 1989). Ein starkes Abmagern als Folge der Mobilisation von Körperreserven beeinflußt das folgende Fruchtbarkeitsgeschehen. Bei Erstlingssauen führt eine unzureichende Versorgung zu einem verlängerten Intervall vom Absetzen bis zur nächsten Rausche (KING und DUNKIN, 1986; KOKETSU et al., 1996). Für Altsauen wurde von KOKETSU et al. (1996) ein Einfluß auf die Wurfgröße des Folgewurfes festgestellt.

Eine indirekte Selektion auf eine verringerte Futteraufnahme hat möglicherweise einen negativen Einfluß auf die Futteraufnahmekapazität von Sauen in der Laktation. Die ermittelten Beziehungen der individuellen Futteraufnahme von Ebern und der Wurfleistung ihrer Nachkommen weisen auf die Möglichkeit hin, die schon früh in Eigenleistungsprüfungen von Ebern gewonnenen Informationen in die Selektionsentscheidung bezüglich der späteren Fruchtbarkeit ihrer Nachkommen einzubeziehen. Sie lassen es auf jeden Fall sinnvoll erscheinen, die tierindividuelle Futteraufnahme in das Zuchtziel von Mutterlinien einzubeziehen, um eine weitere züchterische Reduzierung der Futteraufnahme und der Futteraufnahmekapazität in der Laktation zu vermeiden. Weiterhin gibt es Hinweise, dass neben der mittleren aufgenommenen Futtermenge auch der Futteraufnahmeverlauf die Fruchtbarkeit beeinflußt. In dieser Untersuchung wurden nur Erstwurfinformationen berücksichtigt. Ob ähnliche genetische Korrelationen auch für Sauen höherer Wurfnummer gelten, soll in weiteren Analysen untersucht werden.

\section{Literatur}

CAMERON, N.D.; PEARSON, M.; RICHARDSON, B.; BRADE, M.:

Genetic and phenotypic parameters for performance traits in pigs with ad libitum and restricted feeding. Proc. $4^{\text {th }}$ World Congr. Genet. Appl. Livest. Prod. 15 (1990), 473-476

CARROLL, C.M.; LYNCH, P.B.; BOLAND, M.P.; SPICER, L.J.; AUSTIN, F.H.; LEONARD, N.; ENRIGHT, W.J.; ROCHE, J.F.:

The effects of food intake during lactation and post weaning on the reproductive performance and hormone and metabolite concentrations of primiparous sows. Anim. Sci. 63 (1996), 297-306

CRUMP, R.E.; THOMPSON, R.; HALEY, C.S.; MERCER, J.:

Individual animal model estimates of genetic correlations between performance test and reproduction traits of Landrace pigs performance tested in a commercial nucleus herd. Anim. Sci. 65 (1997), 291298

FISCHER, R.; SPILKE, J.; LENGERKEN, G. v.:

Einbeziehung von Fruchtbarkeitsleistungen in die Zuchtwertschätzung beim Schwein. 1. Mitteilung: Berulcksichtigung aufeinanderfolgender Wurfleistungen einer Sau. Züchtungskunde, Stuttgart 71 (1999), 196-208

GROENEVELD, E.:

VCE Version 4.2.5. Institute of Animal Husbandry and Animal Sciences, Federal Agricultural Research Center (FAL), Mariensee (1998) 


\section{HUGHES, P.E.:}

Nutrition - reproduction interactions in the breeding sow. In: Barnett, J.L.; Hennessy, D.P. (Eds.) Manipulating pig production II. Australasian Pig Science Association, Victoria, (1989), 277-280

JOHANSSON, K.; KENNEDY, B.W.: Genetic and phenotypic relationships of performance test measurements with fertility in Swedish Landrace and Yorkshire sows. Acta Agric. Scand. 33 (1983), 195-199

KERR, J.C.; CAMERON, N.D.:

Genetic and phenotypic relationships between performance test and reproduction traits in pigs selected for components of efficient lean growth rate. Anim. Sci. 60 (1995), 511

KING, R.H.;. DUNKIN, A.C.:

The effect of nutrition on the reproductive performance of first-litter sows: 3 . The response to graded increases in food intake during lactation. Anim. Prod. 42 (1986), 119-125

KOKETSU, Y.; DIAL, G.D.; PETTIGREW, J.E.; MARSH, W.E.; KING, V.L.:

Feed intake pattern during lactation and subsequent reproductive performance of sows. J. Anim. Sci. 74 (1996), 2875-2884

LABROUE, F.; SELLIER, P.; GUEBLEZ, R.; MEUNIER-SALAUN, M.C.:

Estimation des paramètres génétiques pour les critères de comportement alimentaire dans les races Large White et Landrace Français. Journées Recherche Porcine en France 28 (1996), 23-30

MRODE, R.A.; KENNEDY, B.W.:

Genetic variation in measures of food efficiency in pigs and their genetic relationships with growth rate and backfat. Anim. Prod. 56 (1993), 225-232

MULLAN, B.P.; WILLIAMS, I.H.:

The effect of body reserves at farrowing on the reproductive performance of first-litter sows. Anim. Prod. 48 (1989), 449-457

ROEHE, R.; KALM, E.:

Estimation of genetic and environmental risk factors associated with pre-weaning mortality in piglets using generalized linear mixed models. Anim. Sci. 70 (2000), 227-240

ROEHE, R.; KENNEDY, B.W.:

Estimation of genetic parameters for litter size in Canadian Yorkshire and Landrace swine with each parity of farrowing treated as a different trait. J. Anim. Sci. 73 (1995), 2959-2970

RYDHMER, L.; JOHANSSON, K.; STERN, S.; ELIASSON-SELLING, L.:

A genetic study on pubertal age, litter traits, weight loss during lactation and relations to growth and leanness in gilts. Acta Agric. Scand. 42. (1992), 211-219

TÄUBERT, H.; BRANDT, H.:

Varianz- und Kovarianzkomponentenschătzung fur die Wurfleistung von Reinzucht- und Kreuzungssauen. Zuchtungskunde 72 (2000), 43-58

VAN STEENBERGEN, E.J.; KANIS, E.; VAN DER STEHEN, H.A.M.:

Genetic parameters of fattening performance and exterior traits of boars tested in central stations. Livest. Prod. Sci. 24. (1990), 65-82

VON FELDE, A.; ROEHE, R.; LOOFT, H.; KALM, E.:

Genetic association between feed intake and feed intake behaviour at different stages of growth of group-housed boars. Livest. Prod. Sci. 47 (1996), 11-22

Eingegangen: 14.07 .2000

Akzeptiert: 01.09 .2000

Anschriften der Verfasser

SUSANNE KARSTEN, PD Dr. RAINER RÖHE, Prof. Dr. Dr. h.c. mult. ERNST KALM

Christian-Albrechts-Universităt zu Kiel

Institut für Tierzucht und Tierhaltung

Olshausenstr. 40

D-24098 Kiel

Dr. HOLGER LOOFT

PIC Deutschland GmbH

Ratsteich 31

D-24837 Schleswig 
Arch. Tierz., Dummerstorf 43 (2000) 5, 462

\title{
Buchbesprechung
}

\section{Ultrasonography and Reproduction in Swine} Principles and practical applications

\author{
F. MARTINAT-BOTTE, G. RENAUD, F. MADEC, P. COSTIOU, M. TERQUI
}

104 Seiten, zahlreiche Abbildungen, INRA-Editions RD 10, 78026 Versailles cedex, France, 2000, ISBN 2-7380-0887-9, Preis $300 \mathrm{~F}$ (ca. 92,00 DM oder 46 Euro)

INRA-Editions@versailles.inra.fr

Durch die Verfügbarkeit kleinerer und stärkerer Ultraschallsonden in Verbindung mit kleinen tragbaren Ultraschallgeräten werden detaillierte Untersuchungen der verschiedenen Organsysteme auch beim Nutztier unter Praxisbedingungen möglich. Das Buch aus der Reproduktionsforschungsgruppe der INRA in Frankreich hat dazu einen hervorragenden Beitrag in Form dieses reich illustrierten Buches fur das Fortpflanzungssystem beim weiblichen Schwein abgeliefert. Neben den Grundlagen der Ultraschalltechnik und einem kurzen Abriß der Anatomic und Physiologie der Reproduktion werden die Ultraschallbefunde während Brunstzyklus, Trăchtigkeit sowie der post partum und Laktationsphase im Vergleich mit Skizze und anatomischem Bild dargestellt. Weiterhin wird ausführlich auf Abnormalităten und die Anwendung im Herdenmanagement eingegangen. Auch die wichtigsten Artefakte von Ultraschallbildern werden im Detail besprochen und dargestellt, so dass insgesamt auch für den Anfänger eine solide Grundlage für einen Einstieg in diese Technologie gegeben wird. Sehr wertvoll sind die kurzen Zusammenfassungen und Schlußfolgerungen sowie die kurze, aber informative Liste mit weiterfuhrender Literatur am Ende eines jeden Kapitels für ein vertiefendes Studium der jeweiligen Detailaspekte. Das aus dem Französischen ins Englische ubersetzte Buch ist gut lesbar und stellt sowohl für den Anfänger als auch für den Fortgeschrittenen in diesem zukunftsträchtigen Bereich einen wertvollen und deshalb sehr empfehlenswerten Beitrag dar, zumal die Kosten für ein Buch in dieser hohen Qualitătsaufmachung akzeptabel sind. 